\title{
Flavoxate Hydrochloride
}

National Cancer Institute

\section{Source}

National Cancer Institute. Flavoxate Hydrochloride. NCI Thesaurus. Code C47533.

The hydrochloride salt form of flavoxate, a parasympatholytic agent with antispasmodic activity. Flavoxate hydrochloride competitively binds to muscarinic receptors, thereby preventing the actions of acetylcholine. This relaxes vascular smooth muscle, mainly of the urinary tract, and prevents smooth muscle contractions. 